\title{
The pain symptoms and mass recurrence rates after ovarian cystectomy or uni/bilateral oophorectomy procedures in patients over $\mathbf{4 0}$ years old with endometriosis
}

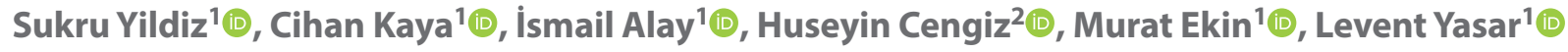 \\ ${ }^{1}$ University of Health Sciences Istanbul, Bakirkoy Dr. Sadi Konuk Educational and Research Hospital, Bakirkoy, Istanbul, Turkey \\ ${ }^{2}$ Istanbul Aydin University, Faculty of Medicine, Istanbul, Turkey
}

\begin{abstract}
Objectives: To evaluate the rates of pain and mass recurrence of the patients over 40 years old with endometriosis who underwent ovarian cystectomy or uni/bilateral oophorectomy.

Material and methods: A retrospective study was conducted with 98 patients undergoing laparoscopic surgery for endometriosis in a tertiary referral center between the time period July 2015 and July 2019. All the patients followed every 3 months and requested to fill the Visual Analogue Scale (VAS) for evaluation of pelvic pain and an ultrasound scan was performed. The inclusion criteria for this study were as follows, patients with ages over 40 , with regular menstrual periods, and who denied hysterectomy and any postoperative hormonal medical treatments.

Results: When the groups were compared in terms of age, body mass index, cyst diameter, CA-125 serum concentrations, preoperative and after surgical pelvic pain scores, mean follow up periods, postoperative hospital stay. However, each of the mean numbers of gravidity and parity were significantly higher than bilateral salpingo-oophorectomy (BSO) groups compared to the other groups ( $p=0.04$ and $p=0.03$, respectively). The laterality, the recurrence rates, and the type of recurrence did not have a significant effect in the group comparison.

Conclusions: The ovarian tissue preserving procedures could be offered for the women over 40 years old suffering from endometriosis with no significant increase in pain symptom or mass recurrence rates considering beneficial effects of estrogen on cardiovascular system, vasomotor symptoms, and bone mineral density.
\end{abstract}

Key words: endometriosis; pain symptom; recurrence; oophorectomy

Ginekologia Polska 2020; 91, 6: 295-300

\section{INTRODUCTION}

Endometriosis is characterized by the existence of endometrial-like tissue outside of the uterine cavity and especially endometriosis causes pelvic pain and infertility during oestrogen-dependent reproductive years. The most affected organ is the ovary, and this affected ovarian tissue is called a chocolate cyst or ovarian endometrioma. Ovarian endometriomas are observed in $17-44 \%$ of women with endometriosis [1].

Laparoscopic conservative surgery is recommended as the main surgical treatment in ovarian endometrioma [2-3]. However, the recurrence of endometrioma after surgery is a major problem. The rate of recurrence is very highly variable and reported with a range between $19 \%$ and $50 \%$ [4]. This recurrence may cause the reappearance of pelvic pain and requiring repeat surgery. The mechanism of recurrence is not fully understood, though it is generally believed to be as follows: the lesion is not removed completely, leading to recurrence from the residual lesion; eutopic endometrial tissue is the key factor in the onset of endometriosis according to the known retrograde menstruation theory [5]. In addition, there is no general agreement in evaluating the conditions that may cause the recurrence of endometrioma.

The age of the patient at the time of the operation was thought to affect the recurrence of endometriosis [6]. Until now, there has been a remarkable inadequacy of attention in the management opportunities for endometriosis patients who are past their reproductive years and in the natural transition towards menopause. However, compared with younger women, women older than 40 years may have more 
dilemmas during management, such as whether to retain ovarian tissue and whether it is necessary to add medical treatment after surgery in those for whom the use of oral contraceptives is no longer recommended [7]. Additionally, new studies of the factors related to postoperative recurrence in women over 40 years of age with endometriosis would help to provide references for the surgical strategy.

In conclusion, our study was aimed to determine the recurrence conditions after cystectomy or uni/bilateral oophorectomy surgeries for ovarian endometrioma in patients aged older than 40 years.

\section{MATERIAL AND METHODS}

All premenopausal women over 40 years of age who experienced laparoscopic surgery for endometrioma were observed at our tertiary referral centre between January 2015 and January 2018.

The inclusion criteria were as follows: there was postoperative histopathological confirmation, patients had regular menstrual periods and denied the hysterectomy procedure and postoperative hormonal treatment, clinical follow-up and pathological data were completed, and patients were classified with stage 3/4 endometriosis patients according to the American Society of Reproductive Medicine (ASRM) classification [8]. The exclusion criteria were hysterectomy; ovarian endometrioma treated with ablation, drainage or incompletely stripping; postmenopausal status; other types of postoperative treatment, such as combined oral contraceptives, progestins, gonadotropin-releasing hormone agonists (GnRHa) or levonorgestrel-releasing intrauterine devices; and a histopathology of malignancy or borderline tumours.

The following data, including age, number of pregnancies, ovarian size $(\mathrm{mm})$, laterality of ovarian cysts, preoperative and postoperative $3^{\text {rd }}$ month visual analogue scales (VAS), body mass indexes, preoperative CA-125 levels, types of laparoscopic surgery, and recurrence during follow up (month) were retrospectively evaluated. Women were each requested for observation three months of the initial year and then annually.

The definition of recurrence of endometrioma was a radiologic appearance of an endometrioma with a circular-form cyst structure (at least $2 \mathrm{~cm}$ in size), surrounded with thick membrane, regularly boarded, and homogenous depleted echogenic substance with dispersed internal echogenic appearance, the absence of papillary structures, and/or pelvic pain [9].

In our study, we performed cystectomy, unilateral salpingo-oophorectomy (USO) and bilateral salpingo-oophorectomy (BSO), and the data from these three groups of patients were analysed.

The surgery was done throughout an umbilical insertion and right and left infra- umbilical insertions by $5 \mathrm{~mm}$ grasping forceps and scissors. Pneumoperitoneum was obtained using a Veress needle (Ethicon Endo-surgery Inc., USA) insertion. In all cases $\mathrm{CO}_{2}$ was used with $12 \mathrm{mmHg}$ intrabdominal pressure. An incision was performed on the endometrioma, and the tissues were dissected, and then exposed. After aspiration of the cyst contents, pathological tissue was pulled from different angles and separated from ovarian parenchyma. Haemostasis was accomplished by bipolar coagulation. Additionally, laparoscopic USO and BSO procedures were performed with the same process and subsequent infundibulopelvic ligament identification and adnexal removal. When the adhesion was observed endometrioma were released from normal tissue by different cleavages. After endometriomas were removed visible endometriotic lesions were excised or coagulated with bipolar energy. Anatomical restoration was then achieved in all cases.

Data analysis was achieved with SPSS (version 20.0; SPSS Inc., Chicago, IL, USA). All data were shown as mean \pm standard deviation. A one-sample Kolmogorov-Smirnov test was accomplished to examine the dispersion of clinical and surgical outcomes. Parametric variables were analyzed by Student's t-test; non-parametric variables were analyzed by Mann-Whitney $U$ test and chi-square test. A p-value of $<0.05$ was determined to be statistically significant for analysis.

\section{RESULTS}

A total of 98 patients who had an adequate after surgical follow-up and met the inclusion criteria were participated in the study. The main groups of study were cystectomy patients $(n=30)$, USO patients $(n=39)$ and BSO patients $(n=29)$. The baseline characteristics are shown in Table 1 . When the groups were compared, there was no significant effect of patient age during the operation. However, the mean numbers of both gravidity and parity were significantly higher in the BSO group compared to the other groups ( $p=0.04$ and $p=0.03$ respectively). There were no significant differences between the groups regarding body mass index, preoperative CA-125 levels, preoperative and postoperative third month VAS scores, the number of previous endometriosis surgeries, ovarian lesion size or postoperative hospital stay. The mean postoperative follow-up was 23.0 months (range 3-34 months) in all women. There were also no significant differences in the recurrence time after surgery between the groups.

The risk factors associated with the endometrioma recurrence are presented in Table 2. There were no differences between the groups regarding the laterality of lesions or the usage of preoperative medication. Additionally, the presence of a pouch of Douglas obliteration did not significantly affect the recurrence rate. 
Table 1. Comparison of the basic characteristics of the study groups

\begin{tabular}{|l|l|l|l|l|}
\hline & Cystectomy $(\mathbf{n}: \mathbf{3 0})$ & USO (n: 39) & BSO (n: 29) & p value \\
\hline Age [years] & $41.86 \pm 3.27$ & $42.64 \pm 3.08$ & $43.34 \pm 3.16$ & 0.1 \\
\hline Gravidity & $1.36 \pm 1.15$ & $1.58 \pm 0.71$ & $1.93 \pm 1.09^{\mathrm{a}, \mathrm{b}, \mathrm{c}}$ & $\mathbf{0 . 0 4}$ \\
\hline Parity & $1.31 \pm 1.11$ & $1.55 \pm 0.69$ & $1.82 \pm 1.1^{\mathrm{d}, \mathrm{e}, \mathrm{f}}$ & $\mathbf{0 . 0 3}$ \\
\hline BMI [kg/m & $24.14 \pm 2.35$ & $25.89 \pm 3.57$ & $26.23 \pm 4.27$ & 0.07 \\
\hline Size [mm] & $70.3 \pm 34.83$ & $66.43 \pm 26.33$ & $53.85 \pm 23.07$ & 0.27 \\
\hline CA-125 IU/mL & $85.43 \pm 129.32$ & $96.49 \pm 181.64$ & $73.36 \pm 68.03$ & 0.66 \\
\hline Pre-op Pelvic pain [VAS] & $8(0-10)$ & $8(0-10)$ & $8(0-10)$ & 0.99 \\
\hline Post-op 3'd month Pelvic pain [VAS] & $3.5(0-7)$ & $4(0-8)$ & $3(0-9)$ & 0.61 \\
\hline Number of previous endometriosis surgeries & $0.16 \pm 0.46$ & $0.23 \pm 0.58$ & $0.13 \pm 0.35$ & 0.83 \\
\hline Mean follow-up [months] & $24.26 \pm 10.49$ & $22.10 \pm 10.75$ & $22.51 \pm 9.77$ & 0.51 \\
\hline Hospital stay [days] & $3.63 \pm 1.71$ & $3.23 \pm 1.11$ & $3.96 \pm 2$ & 0.40 \\
\hline Recurrence time [months] & $2.83 \pm 7.79$ & $2.61 \pm 8.11$ & $0.93 \pm 4.47$ & 0.69 \\
\hline A pval
\end{tabular}

A p value of $<0.05$ was considered significant for all bold values; USO — unilateral salpingo-oophorectomy; BSO — bilateral salpingo-oophorectomy; VAS — visual analogue scale; BMI — body mass index; ${ }^{a, d}-p<0.001$ (cystectomy vs USO), b,e $-p<0.001$ (cystectomy vs BSO), ${ }^{c, f}-p<0.001$ (USO vs BSO)

\begin{tabular}{|c|c|c|c|c|c|}
\hline & & Cystectomy & USO & BSO & p value \\
\hline \multirow{2}{*}{ Laterality } & Unilateral & $21(70 \%)$ & 24 (61.5\%) & 13 (44.8\%) & \multirow{2}{*}{0.06} \\
\hline & Bilateral & $9(30 \%)$ & $15(38.5 \%)$ & $16(55.2 \%)$ & \\
\hline \multirow{2}{*}{$\begin{array}{l}\text { Preoperative } \\
\text { medication }\end{array}$} & Yes & $8(26.7 \%)$ & $11(28.2 \%)$ & $7(24.1 \%)$ & \multirow{2}{*}{0.82} \\
\hline & No & $22(73.3 \%)$ & $28(71.8 \%)$ & 22 (75.9\%) & \\
\hline \multirow{2}{*}{ Douglas obliteration } & Yes & $22(73.3 \%)$ & 28 (71.8\%) & 18 (62.1\%) & \multirow{2}{*}{0.35} \\
\hline & No & $8(26.2 \%)$ & $11(28.2 \%)$ & $11(37.9 \%)$ & \\
\hline \multirow{2}{*}{ Recurrence rate } & Yes & $4(13.3 \%)$ & $4(10.3 \%)$ & $2(6.9 \%)$ & \multirow{2}{*}{0.41} \\
\hline & No & $26(86.7 \%)$ & 35 (89.7\%) & 27 (93.1\%) & \\
\hline \multirow{3}{*}{ Type of recurrence } & Pain & $2(6.7 \%)$ & $3(7.7 \%)$ & $2(6.9 \%)$ & \multirow{3}{*}{0.58} \\
\hline & Mass & $2(6.7 \%)$ & $1(2.6 \%)$ & 0 & \\
\hline & None & 26 (86.7\%) & 35 (89.7\%) & 27 (93.1\%) & \\
\hline
\end{tabular}

USO - unilateral salpingo-oophorectomy; BSO - bilateral salpingo-oophorectomy; a p value of $<0.05$ was considered significant for all bold values

The recurrence rate following endometrioma surgery was $13.3 \%$ in the cystectomy group, $10.3 \%$ in the USO group and $6.9 \%$ in the BSO group. There were no statistically significant differences in the recurrence rate between the groups. Additionally, a type of recurrence analysis was conducted between the cystectomy group (pain $6.7 \%$, mass $6.7 \%$ ), USO group (pain $7.7 \%$, mass $2.6 \%$ ), and BSO group (pain $6.9 \%$, mass $0 \%$ ); and there were no statistically significant difference within these groups.

\section{DISCUSSION}

This study evaluated the rates of pain and mass recurrence in patients over 40 years old with endometriosis who underwent ovarian cystectomy or uni/bilateral oophorectomy surgeries. The recurrence rate reported in the literature varies significantly from $6 \%$ to $67 \%$ due to dif- ferent follow-up durations, different surgical techniques and different diagnostic criteria [11-12]. Age-dependent endometriosis data have demonstrated that a younger age during the endometriosis operation is related with recurrence of endometrioma [13-14]. Another study showed that endometrioma recurrence was higher in patients undergoing an endometriosis operation under the age of 32 [15]. In younger women, endometriomas may be more aggressive due to a high level of hormones, consequently it may cause more recurrence rates [15-16]. Additionally, a study showed that endometriosis might develop from follicles or the corpus luteum [17]. On the other hand, this issue was discussed for the first time in 1993 by Witt and Barad with a study proposed at discussing the treatment modalities in women with an endometrioma above 40 years of age [18]. 
Oestrogen plays important roles in processes that protect heart and bone physiology [19]. Along with these significant effects of oestrogen, the physical changes during the perimenopausal years are established in hormonal alterations, particularly variations in the level of circulating oestrogen. This premenopausal population faces extraordinary problems while managing the effects of endometriosis on the patient. Some of these comprehended the elevated risk of medical contraindications to combined hormonal contraceptives (COC) and prolonged disease significantly increases the risk of cancer, with the possibility of causing malignant transformation [20]. Because of this condition, and considering the different types of laparoscopic surgeries, the management of oestrogen-dependent diseases such as endometriosis may become complicated in women over 40 years old due to the severe alterations of oestrogen levels. According to these data, our study is focused on determining the rate of recurrence in patients over 40 years old with endometriosis who underwent different type of laparoscopic surgeries.

Variations in recommended recurrence criteria, follow-up time, and study populations may result in different rates of recurrence rates of endometrioma in the literature [21]. Chan et al. [22] founded that dysmenorrhea and septated ovarian cysts significantly altered the recurrence rate after surgery. Additionally, a study showed that the depth of invasion of the endometrial tissue into the normal tissue was an independent risk factor for recurrence rate of endometrioma [23]. Another study showed that CA-125 serum concentrations, cyst diameter and previous pelvic surgery would affect the recurrence rate [24]. Our study showed no significant association between recurrence rate and patient-related factors such as gravidity, parity, preoperative serum CA-125 level, body mass index and cyst diameter. Additionally, Campo et al. [25] investigated that the relationship between endometrioma recurrence and surgical situations such as mass size, number and laterality, adhesions or implantations, existence of spillage and demographic situations such as age, body mass index, family history; the authors showed that family history of endometriosis was the only factor affecting endometrioma recurrence rate. On the other hand, another study suggested that the cumulative recurrence rate of ovarian endometrioma was related with the since onset of time follow-up and severe endometriosis, as the second-line surgery seemed to be a risk factor associated with the increased recurrence rate [26]. Previous studies concluded that the cumulative recurrence rates of conservative surgery, semi-radical surgery and radical surgery were $28.9 \%, 17.3 \%$ and $0 \%$ respectively [27]. In our study, the cumulative recurrence rates in the cystectomy, USO and BSO surgery group were $13.3 \%, 10.3 \%$ and $6.9 \%$, respectively.
The international guidelines on endometrioma have not recommended exact suggestions for the management of patient approaching menopause, likely due to the lack of evidence. The 2014 European Society of Human Reproduction and Embryology guidelines suggest considering definitive operation (hysterectomy with bilateral oophorectomy, together with the removal of other lesions) for women who terminate their reproductive life and had persisting symptoms despite medical therapy [28]. The guidelines also proposed to brief the women in whom hysterectomy would not necessarily treat endometriosis symptoms. Alike recommendations have been suggested by the Society of Obstetricians and Gynecology of Canada [29]. In addition, the World Endometriosis Society consensus on the present treatment of endometriosis has announced that suggestions were associated to women of reproductive age but emphasized that the role of hysterectomy and simultaneous oophorectomy demonstrates the ensuring a little reliable evidence effect [30]. On the other hand, in recent years, an increasing number of patients have expected to preserve the ovaries and the uterus. All patients included in our study denied hysterectomy procedure. Additionally, clinicians pay more attention to the preservation of reproductive organs in perimenopausal patients due to the beneficial effects of oestrogen levels. Furthermore, additional operations may increase the duration of surgery and morbidity. Zing et al. [7] showed that the ovarian preservation surgery was an independent risk factor for recurrence in patients with ovarian endometriosis (> 45 year old). However, our results showed no significant difference in recurrence rate with different types of endometrioma surgeries.

In different studies evaluating the pathophysiology of recurrence, it has been suggested that the recurrent lesion may derived from remaining tissue. Failure to completely remove pathological tissues at the time of primary surgery may increase the likelihood of recurrence to endometrioma, and the larger part of recurrent lesions were formed in the same area [31-32]. Thus, during the primary surgery for endometriosis, an excellent surgical technique and the experienced endometrioma surgeons are critical due to the capability of pathologic residual tissue to grow.

For many years, the medications used in the management of endometriosis included selective oestrogen receptor modulators, aromatase inhibitors, $\mathrm{GnRHa}$, selective progesterone receptor modulators and angiogenic agents. A Cochrane review on the use of postoperative medication in endometriosis suggested that there was no evidence of any improved pain relief, though the level of evidence was not sufficient in the reported literature [5]. However, A study found that use of GnRHa after surgery could only extend the recurrence interval but not decrease the whole recurrence rate [33]. In our study, endometrioma 
management included only surgery without the addition of postoperative medications.

However, our study also has a few limitations. First the study was not a randomized controlled study; therefore, some possible biases could have occurred. In addition, our study focused on different types of adnexal surgeries, and hysterectomy operations could be added for the analysis. Additionally, patients with histopathology of malignancy or borderline tumours were excluded from the study due to the requirement of oncologic management. This situation may cause additional limitation in our study. Although endometriosis-associated malignant ovarian tumours are usually early stage, oncologic safety of the surgical procedures such as cystectomy could not be analyzed due to this limitation [34]. Finally, this study had a relatively small sample size.

In conclusion, the rate and prevention of disease recurrence in women approaching menopause are unknown factors. Our study showed that ovarian tissue-preserving procedures could be offered for the women over 40 years old suffering from endometriosis with no significant increase in pain symptoms or mass recurrence rates considering the beneficial effect of oestrogen on the cardiovascular system, vasomotor symptoms and bone mineral density.

\section{REFERENCES}

1. Busacca M, Vignali M. Ovarian endometriosis: from pathogenesis to surgical treatment. Curr Opin Obstet Gynecol. 2003; 15(4): 321-326, doi: 10.1097/01.gco.0000084247.09900.4f, indexed in Pubmed: 12858105.

2. Chapron $C$, Vercellini $P$, Barakat $H$, et al. Management of ovarian endometriomas. Hum Reprod Update. 2002; 8(6): 591-597, doi: 10.1093/humupd/8.6.591, indexed in Pubmed: 12498427.

3. Hart RJ, Hickey $M$, Maouris $P$, et al. Excisional surgery versus ablative surgery for ovarian endometriomata. Cochrane Database Syst Rev. 2005(3): CD004992, doi: 10.1002/14651858.CD004992.pub2, indexed in Pubmed: 16034960.

4. Busacca M, Marana R, Caruana P, et al. Recurrence of ovarian endometrioma after laparoscopic excision. American Journal of Obstetrics and Gynecology. 1999; 180(3): 519-523, doi: 10.1016/s0002-9378(99)70247-4.

5. Canis M, Bourdel N, Houlle C, et al. Endometriosis may not be a chronic disease: an alternative theory offering more optimistic prospects for our patients. Fertil Steril. 2016; 105(1): 32-34, doi: 10.1016/j.fertnstert.2015.09.009, indexed in Pubmed: 26453981.

6. Kikuchi I, Takeuchi H, Kitade $M$, et al. Recurrence rate of endometriomas following a laparoscopic cystectomy. Acta Obstet Gynecol Scand. 2006; 85(9): 1120-1124, doi: 10.1080/00016340600627154, indexed in Pubmed: 16929419.

7. He ZX, Sun TT, Wang S, et al. Risk Factors for Recurrence of Ovarian Endometriosis in Chinese Patients Aged 45 and Over. Chin Med J (Engl). 2018; 131(11): 1308-1313, doi: 10.4103/0366-6999.232790, indexed in Pubmed: 29786043.

8. Revised American Society for Reproductive Medicine classification of endometriosis: 1996 . Fertility and Sterility. 1997; 67(5): 817-821, doi: 10.1016/s0015-0282(97)81391-x.

9. Mais V, Guerriero S, Ajossa S, et al. The efficiency of transvaginal ultrasonography in the diagnosis of endometrioma. Fertility and Sterility. 1993; 60(5): 776-780, doi: 10.1016/s0015-0282(16)56275-x.

10. Kennedy S, Bergqvist A, Chapron C, et al. ESHRE Special Interest Group for Endometriosis and Endometrium Guideline Development Group. ESHRE guideline for the diagnosis and treatment of endometriosis. Hum Reprod. 2005; 20(10): 2698-2704, doi: 10.1093/humrep/dei135, indexed in Pubmed: 15980014
11. Morgante G, Ditto A, La Marca A, et al. Low-dose danazol after combined surgical and medical therapy reduces the incidence of pelvic pain in women with moderate and severe endometriosis. Hum Reprod. 1999; 14(9): 2371-2374, doi: 10.1093/humrep/14.9.2371, indexed in Pubmed: 10469713.

12. Vignali $M$, Bianchi $S$, Candiani $M$, et al. Surgical treatment of deep endometriosis and risk of recurrence. J Minim Invasive Gynecol. 2005; 12(6): 508-513, doi: 10.1016/j.jmig.2005.06.016, indexed in Pubmed: 16337578.

13. Liu X, Yuan L, Shen F, et al. Patterns of and risk factors for recurrence in women with ovarian endometriomas. Obstet Gynecol. 2007; 109(6): 1411-1420, doi: 10.1097/01.AOG.0000265215.87717.8b, indexed in Pubmed: 17540815.

14. $\mathrm{Li} \mathrm{Hj}$, Leng Jh, Lang Jh, et al. [Correlative factors analysis of recurrence of endometriosis after conservative surgery]. Zhonghua Fu Chan Ke Za Zhi. 2005; 40(1): 13-16, indexed in Pubmed: 15774085.

15. Ouchi N, Akira S, Mine K, et al. Recurrence of ovarian endometrioma after laparoscopic excision: risk factors and prevention. J Obstet Gynaecol Res. 2014; 40(1): 230-236, doi: 10.1111/jog.12164, indexed in Pubmed: 24102958.

16. Sengoku K, Miyamoto T, Horikawa M, et al. Clinicopathologic risk factors for recurrence of ovarian endometrioma following laparoscopic cystectomy. Acta Obstet Gynecol Scand. 2013; 92(3): 278-284, doi: 10.1111/aogs.12051, indexed in Pubmed: 23194011.

17. Vercellini $P$, Somigliana $E$, Vigano $P$, et al. 'Blood On The Tracks' from corpora lutea to endometriomas. BJOG. 2009; 116(3): 366-371, doi: 10.1111/j.1471-0528.2008.02055.x, indexed in Pubmed: 19187368.

18. Witt BR, Barad DH. Management of endometriosis in women elder than 40 years of age. Obstet Gynecol Clin North Am. 1993; 20(2): 349-363, indexed in Pubmed: 8367137.

19. Hou H, Zhao Z, Machuki JO, et al. Estrogen deficiency compromised the $\beta A R-G s / G i$ coupling: implications for arrhythmia and cardiac injury. Pflugers Arch. 2018; 470(3): 559-570, doi: 10.1007/s00424-017-2098-4, indexed in Pubmed: 29297096.

20. Vercellini $P$, Viganò $P$, Buggio $L$, et al. Perimenopausal management of ovarian endometriosis and associated cancer risk: When is medical or surgical treatment indicated? Best Pract Res Clin Obstet Gynaecol. 2018; 51: 151-168, doi: 10.1016/j.bpobgyn.2018.01.017, indexed in Pubmed: 29551389.

21. Sesti F, CapozzoloT, Pietropolli A, et al. Recurrence rate of endometrioma after laparoscopic cystectomy: a comparative randomized trial between post-operative hormonal suppression treatment or dietary therapy vs. placebo. Eur J Obstet Gynecol Reprod Biol. 2009; 147(1): 72-77, doi: 10.1016/j.ejogrb.2009.07.003, indexed in Pubmed: 19665279.

22. Chon SJ, Lee $\mathrm{SH}$, Choi $\mathrm{JH}$, et al. Preoperative risk factors in recurrent endometrioma after primary conservative surgery. Obstet Gynecol Sci. 2016; 59(4): 286-294, doi: 10.5468/ogs.2016.59.4.286, indexed in Pubmed: 27462595

23. Selcuk S, Cam C, Koc N, et al. Evaluation of risk factors for the recurrence of ovarian endometriomas. Eur J Obstet Gynecol Reprod Biol. 2016; 203: 56-60, doi: 10.1016/j.ejogrb.2016.05.008, indexed in Pubmed: 27240262.

24. Guzel Al, Topcu HO, Ekilinc S, et al. Recurrence factors in women underwent laparoscopic surgery for endometrioma. Minerva Chir. 2014;69(5): 277-282, indexed in Pubmed: 25267018.

25. Campo S, CampoV, Gambadauro P. Is a positive family history of endometriosis a risk factor for endometrioma recurrence after laparoscopic surgery? Reprod Sci. 2014; 21(4):526-531, doi: 10.1177/1933719113503413, indexed in Pubmed: 24026309.

26. Kim ML, Kim JM, Seong SJu, et al. Recurrence of ovarian endometrioma after second-line, conservative, laparoscopic cyst enucleation. Am J Obstet Gynecol. 2014; 210(3): 216.e1-216.e6, doi: 10.1016/j. ajog.2013.11.007, indexed in Pubmed: 24215855.

27. Yang $X \mathrm{H}, \mathrm{Ai} X Z$, Ding $Y$. The research progression of the related factor for endometriosis recurrence. Chin Obstet Gynecol. 2012; 39: 437-445.

28. Dunselman GAJ, Vermeulen N, Becker C, et al. European Society of Human Reproduction and Embryology. ESHRE guideline: management of women with endometriosis. Hum Reprod. 2014; 29(3): 400-412, doi: 10.1093/humrep/det457, indexed in Pubmed: 24435778.

29. Leyland N, Casper R, Laberge P, et al. Endometriosis: Diagnosis and Management. J Obstet Gynaecol Can. 2010; 32(7): 51-53, indexed in Pubmed: 21545757.

30. National Institute for Health and Care Exellence. Endometriosis diagnosis and management (NICE guideline 73). 2017. 
31. Exacoustos $C$, Zupi E, Amadio A, et al. Recurrence of endometriomas after laparoscopic removal: sonographic and clinical follow-up and indication for second surgery. J Minim Invasive Gynecol. 2006; 13(4): 281-288, doi: 10.1016/j.jmig.2006.03.002, indexed in Pubmed: 16825067.

32. Alio L, Angioni S, Arena S, et al. Endometriosis: seeking optimal management in women approaching menopause. Climacteric. 2019; 22(4): 329-338, doi: 10.1080/13697137.2018.1549213, indexed in Pubmed: 30628469.
33. Vercellini P, Crosignani PG, Fadini R, et al. A gonadotrophin-releasing hormone agonist compared with expectant management after conservative surgery for symptomatic endometriosis. Br J Obstet Gynaecol. 1999; 106(7): 672-677, doi: 10.1111/j.1471-0528.1999.tb08366.x, indexed in Pubmed: 10428523.

34. Oral E, Aydin O, Kumbak BA, et al. Concomitant endometriosis in malignant and borderline ovarian tumours. J Obstet Gynaecol. 2018; 38(8): 1104-1109, doi: 10.1080/01443615.2018.1441815, indexed in Pubmed: 29884083. 